\title{
Limit cycles for perturbing Hamiltonian system inside piecewise smooth polynomial differential system
}

Kuilin $\mathrm{Wu}^{1,2}$ and Shimin $\mathrm{Li}^{3^{*}}$

"Correspondence:

lism1983@126.com

${ }^{3}$ School of Mathematics and

Statistics, Guangdong University of

Finance and Economics,

Guangzhou, 510320, P.R. China

Full list of author information is

available at the end of the article

\section{Springer}

\begin{abstract}
In this paper, we obtain the first-order Melnikov function of piecewise smooth polynomial perturbation of a Hamiltonian system. As application, we consider the number of limit cycles for perturbing the global center and truncated pendulum inside a piecewise smooth cubic polynomial differential system. Our results show that a piecewise smooth differential system can bifurcate more limit cycles than the smooth one.
\end{abstract}

MSC: 34A36; 34C07; 37G15

Keywords: limit cycle; Hamiltonian system; piecewise smooth differential system; Melnikov method

\section{Introduction and statement of the main results}

The second part of Hilbert's 16th problem and its weak version are two open problems in the qualitative theory of real planar differential systems; see [1-4]. Since both problems are difficult, mathematicians try to study particular and simple cases. For example, Smale's 13th problem restricts Hilbert's 16th problem to the Liénard systems [5].

In recent years, stimulated by nonsmooth phenomena in the real world, piecewise smooth differential systems have attracted a good deal of attention; see, for instance, [6, 7]. There are several papers $[8,9]$ considering the limit cycles for piecewise smooth Liénard systems. The authors of [10] studied the Hopf bifurcation for a piecewise smooth planar Hamiltonian system. In the paper [11], the authors considered the Poincare bifurcation for piecewise smooth Hamiltonian systems and obtained the first-order Melnikov function. Then, they applied the first-order Melnikov function to study the number of limit cycles that bifurcate from the period annulus of the center and obtained some new results. Later, by introducing multiple parameters, in [12], some new formulas are obtained for piecewise smooth systems. In the paper [13], the authors considered the number of limit cycles that bifurcate from the period annulus of the center for a piecewise smooth quadratic isochronous center. Generally speaking, a piecewise smooth system can bifurcate more limit cycles than a smooth one. In a recent paper [9], the authors studied the limit cycles for $m$-piecewise smooth Liénard system, They conjecture that the number of limit cycles decreases as $m$ increases.

(c) 2016 Wu and Li. This article is distributed under the terms of the Creative Commons Attribution 4.0 International License (http://creativecommons.org/licenses/by/4.0/), which permits unrestricted use, distribution, and reproduction in any medium, provided you give appropriate credit to the original author(s) and the source, provide a link to the Creative Commons license, and indicate if changes were made. 
In this paper, we consider the following piecewise smooth perturbed Hamiltonian system:

$$
\left(\begin{array}{l}
\frac{d x}{d t} \\
\frac{d y}{d t}
\end{array}\right)=\left\{\begin{array}{cc}
\left(\begin{array}{c}
H_{y}(x, y)+\varepsilon f^{+}(x, y) \\
-H_{x}(x, y)+\varepsilon g^{+}(x, y)
\end{array}\right), & x>0, \\
\left(\begin{array}{c}
H_{y}(x, y)+\varepsilon f^{-}(x, y) \\
-H_{x}(x, y)+\varepsilon g^{-}(x, y)
\end{array}\right), & x<0,
\end{array}\right.
$$

where $f^{ \pm}(x, y)$ and $g^{ \pm}(x, y)$ are polynomial functions with respect to $x$ and $y$.

Note that the unperturbed system $\left.(1)\right|_{\varepsilon=0}$ is a Hamiltonian system with the first integral $H(x, y)$, and there is a family of periodic orbits $L_{h}=L_{h}^{+} \cup L_{h}^{-}$surrounding the center, where $L_{h}^{ \pm}: H(x, y)=h, x>0(x<0), h \in\left(h_{1}, h_{2}\right)$. Without loss of generality, we suppose that $L_{h}$ has the clockwise orientation.

In this paper, we try to study the first-order Melnikov function for piecewise smooth system (1). Applying the first-order Melnikov function, we consider the number of limit cycles that bifurcate from the period annulus of the center for unperturbed system (1) $\left.\right|_{\varepsilon=0}$ under piecewise smooth polynomial perturbation.

Theorem 1 The first-order Melnikov function of system (1) can be expressed as

$$
\begin{aligned}
M_{1}(h)= & -\int_{L_{h}^{+}}\left(\int_{0}^{x} g_{y}^{+}(x, y) \mathrm{d} x+f^{+}(x, y)\right) \mathrm{d} y \\
& -\int_{L_{h}^{-}}\left(\int_{0}^{x} g_{y}^{-}(x, y) \mathrm{d} x+f^{-}(x, y)\right) \mathrm{d} y,
\end{aligned}
$$

where $L^{ \pm}(h): H(x, y)=h, x>0(x<0), h \in\left(h_{1}, h_{2}\right)$, and $L_{h}=L_{h}^{+} \cup L_{h}^{-}$has the clockwise orientation.

Moreover, if $M_{1}\left(h^{*}\right)=0$ and $M_{1}^{\prime}\left(h^{*}\right) \neq 0$ for some $h^{*} \in\left(h_{1}, h_{2}\right)$, then for $|\varepsilon|>0$ sufficiently small, system (1) has a unique limit cycle near $L_{h^{*}}$.

Remark 2 If $f^{+}(x, y) \equiv f^{-}(x, y)$ and $g^{+}(x, y) \equiv g^{-}(x, y)$, then system (1) is a smooth nearHamiltonian system, and the first-order Melnikov function is well known; see [3]. Let $\operatorname{deg} H(x, y)=m$. There are many papers considering the number of limit cycles that bifurcate from the period annulus of the center. See, for instance, $m=2$ (e.g. [14]), $m=3$ (e.g. [15]), $m=4$ (e.g. [16]), $m=5$ (e.g. [17, 18]), $m=6$ (e.g. [19]).

As applications, we study the number of limit cycles for the piecewise smooth perturbation of the global center

$$
\left(\begin{array}{c}
\frac{d x}{d t} \\
\frac{d y}{d t}
\end{array}\right)=\left\{\begin{array}{cc}
\left(\begin{array}{c}
y+y^{3}+\varepsilon f^{+}(x, y) \\
-x+\varepsilon g^{+}(x, y)
\end{array}\right), & x>0 \\
\left(\begin{array}{c}
y+y^{3}+\varepsilon f^{-}(x, y) \\
-x+\varepsilon g^{-}(x, y)
\end{array}\right), & x<0
\end{array}\right.
$$

and the truncated pendulum

$$
\left(\begin{array}{c}
\frac{d x}{d t} \\
\frac{d y}{d t}
\end{array}\right)= \begin{cases}\left(\begin{array}{c}
y-y^{3}+\varepsilon f^{+}(x, y) \\
-x+\varepsilon g^{+}(x, y)
\end{array}\right), & x>0 \\
\left(\begin{array}{c}
y-y^{3}+\varepsilon f^{-}(x, y) \\
-x+\varepsilon g^{-}(x, y)
\end{array}\right), & x<0\end{cases}
$$


where

$$
\begin{aligned}
& f^{+}(x, y)=a_{1} x+a_{2} y+a_{3} x^{2}+a_{4} x y+a_{5} y^{2}+a_{6} x^{3}+a_{7} x^{2} y+a_{8} x y^{2}+a_{9} y^{3}, \\
& g^{+}(x, y)=b_{1} x+b_{2} y+b_{3} x^{2}+b_{4} x y+b_{5} y^{2}+b_{6} x^{3}+b_{7} x^{2} y+b_{8} x y^{2}+b_{9} y^{3}, \\
& f^{-}(x, y)=c_{1} x+c_{2} y+c_{3} x^{2}+c_{4} x y+c_{5} y^{2}+c_{6} x^{3}+c_{7} x^{2} y+c_{8} x y^{2}+c_{9} y^{3}, \\
& g^{-}(x, y)=d_{1} x+d_{2} y+d_{3} x^{2}+d_{4} x y+d_{5} y^{2}+d_{6} x^{3}+d_{7} x^{2} y+d_{8} x y^{2}+d_{9} y^{3} .
\end{aligned}
$$

Note that for $\varepsilon=0$, systems (3) and (4) are Hamiltonian systems. These systems occur in oscillating chemical reactor models and have been studied in several papers; see, for instance, $[14,20]$.

Applying the first-order Melnikov function given by (2), we consider the number of limit cycles that can bifurcate from the period annuls surrounding the origin of systems (3) and (4) under piecewise smooth cubic polynomial perturbation. Our result is the following theorem.

Theorem 3 There are at least five limit cycles that can bifurcate from the period annulus surrounding the origin of (3) (resp. (4)) by the first-order Melnikov function.

Remark 4 If $a_{i}=c_{i}, b_{i}=d_{i}, i=1,2, \ldots, 9$, then systems (3) and (4) become smooth nearHamiltonian systems and have been studied in the papers [14,20], where the authors obtained that there are at most two limit cycles that bifurcate from the period annulus of the origin for the smooth systems (3) and (4). Our result shows that planar piecewise smooth differential systems (3) and (4) can bifurcate three more limit cycles than the smooth one.

\section{Proof of Theorem 1}

We need the following lemma derived from [11] to prove Theorem 1.

Lemma 5 Consider the perturbed piecewise smooth Hamiltonian system

$$
\left(\begin{array}{c}
\frac{d x}{d t} \\
\frac{d y}{d t}
\end{array}\right)=\left\{\begin{array}{cc}
\left(\begin{array}{c}
H_{y}^{+}(x, y)+\varepsilon f^{+}(x, y) \\
-H_{x}^{+}(x, y)+\varepsilon g^{+}(x, y)
\end{array}\right), & x>0, \\
\left(\begin{array}{c}
H_{y}^{-}(x, y)+\varepsilon f^{-}(x, y) \\
-H_{x}^{-}(x, y)+\varepsilon g^{-}(x, y)
\end{array}\right), & x<0,
\end{array}\right.
$$

where $f^{ \pm}(x, y)$ and $g^{ \pm}(x, y)$ are analytic functions with respect to $x, y$. Assume that:

(I) There exist an interval $J=(\alpha, \beta)$ and two points $A(h)=(0, \alpha(h))$ and $A_{1}(h)=\left(0, \alpha_{1}(h)\right)$, where $\alpha(h) \neq \alpha_{1}(h)$, such that, for $h \in J$, we have

$$
\begin{aligned}
& H^{+}(A(h))=H^{+}\left(A_{1}(h)\right)=h, \\
& H^{-}(A(h))=H^{-}\left(A_{1}(h)\right) .
\end{aligned}
$$

(II) The system has an orbital arc $L_{h}^{+}$starting from $A(h)$ and ending at $A_{1}(h)$ defined by $H^{+}(x, y)=h, x>0$. The system has an orbital arc $L_{h}^{-}$starting from $A_{1}(h)$ and ending at $A(h)$ defined by $H^{-}(x, y)=h, x \leq 0$.

Under assumptions (I) and (II), system (6) $\left.\right|_{\varepsilon=0}$ has a family of periodic orbits $L_{h}=L_{h}^{+} \cup L_{h}^{-}$ for $h \in J$. Each of the closed curves $L_{h}$ is piecewise smooth in general. Further, without loss of generality, suppose that $L_{h}$ has a clockwise orientation. 
Then the first-order Melnikov function of system (6) can be expressed as

$$
M_{1}(h)=\frac{H_{y}^{+}(A)}{H_{y}^{-}(A)}\left(\frac{H_{y}^{-}\left(A_{1}\right)}{H_{y}^{+}\left(A_{1}\right)} \int_{L_{h}^{+}} g^{+} \mathrm{d} x-f^{+} \mathrm{d} y+\int_{L_{h}^{-}} g^{-} \mathrm{d} x-f^{-} \mathrm{d} y\right) .
$$

Further, if $M_{1}\left(h^{*}\right)=0$ and $M_{1}^{\prime}\left(h^{*}\right) \neq 0$ for some $h^{*} \in J$, then for $|\varepsilon|>0$ sufficiently small, system (6) has a unique limit cycle near $L_{h^{*}}$.

Proof of Theorem 1 Since the unperturbed system $\left.(1)\right|_{\varepsilon=0}$ is a Hamiltonian system with first integral $H(x, y)$, it is obvious that assumptions (I) and (II) are satisfied. Note that $H^{+}(x, y) \equiv$ $H^{-}(x, y)=H(x, y)$ for system (1). Then

$$
H_{y}^{+}(A(h))=H_{y}^{-}(A(h)), \quad H_{y}^{+}\left(A_{1}(h)\right)=H_{y}^{-}\left(A_{1}(h)\right) .
$$

Replacing (9) by (8), we have

$$
M_{1}(h)=M^{+}(h)+M^{-}(h),
$$

where

$$
\begin{aligned}
& M^{+}(h)=\int_{L_{h}^{+}} g^{+}(x, y) \mathrm{d} x-f^{+}(x, y) \mathrm{d} y, \\
& M^{-}(h)=\int_{L_{h}^{-}} g^{-}(x, y) \mathrm{d} x-f^{-}(x, y) \mathrm{d} y .
\end{aligned}
$$

Applying Green's formula two times to the integrals (11), we have

$$
\begin{aligned}
M^{+}(h) & =\int_{L_{h}^{+}} g^{+}(x, y) \mathrm{d} x-f^{+}(x, y) \mathrm{d} y \\
& =\int_{L_{h}^{+}} g^{+}(x, y) \mathrm{d} x-\int_{L_{h}^{+}} f^{+}(x, y) \mathrm{d} y \\
& =\oint_{L_{h}^{+} \cup \overrightarrow{A_{1} A}} g^{+}(x, y) \mathrm{d} x-\int_{L_{h}^{+}} f^{+}(x, y) \mathrm{d} y \\
& =\iint_{\operatorname{int}\left(L_{h}^{+} \cup \overrightarrow{A_{1} A}\right)} g_{y}^{+}(x, y) \mathrm{d} x \mathrm{~d} y-\int_{L_{h}^{+}} f^{+}(x, y) \mathrm{d} y \\
& =\oint_{L_{h}^{+} \cup \overrightarrow{A_{1} A}}\left(\int_{0}^{x} g_{y}^{+}(x, y) \mathrm{d} x\right) \mathrm{d} y-\int_{L_{h}^{+}} f^{+}(x, y) \mathrm{d} y \\
& =-\int_{L_{h}^{+}}\left(\int_{0}^{x} g_{y}^{+}(x, y) \mathrm{d} x+f^{+}(x, y)\right) \mathrm{d} y,
\end{aligned}
$$

where $L_{h}^{+}: H(x, y)=h, x>0$.

Similarly, we have

$$
M^{-}(h)=-\int_{L_{h}^{-}}\left(\int_{0}^{x} g_{y}^{-}(x, y) \mathrm{d} x+f^{-}(x, y)\right) \mathrm{d} y,
$$

where $L_{h}^{-}: H(x, y)=h, x<0$. 
Replacing (12) and (13) by (10), we obtain the first-order Melnikov function (2). According to Lemma 5, every simple zero of the first-order Melnikov function (2) provides a limit cycle of system (1). This completes the proof.

\section{Proof of Theorem 3}

In order to estimate the number of the zeros of the first-order Melnikov function, we need the following lemma.

Lemma 6 [21] Consider $p+1$ linearly independent analytical functions $f_{i}: U \rightarrow \mathbb{R}, i=$ $0,1, \ldots, p$, where $U \in \mathbb{R}$ is an interval. Suppose that there exists $j \in\{0,1, \ldots, p\}$ such that $f_{j}$ has constant sign. Then there exist $p+1$ constants $C_{i}, i=0,1, \ldots, p$, such that $f(r)=$ $\sum_{i=0}^{p} C_{i} f_{i}(r)$ has at least $p$ simple zeros in $U$.

Proof of Theorem 3 First, we consider system (3). From

$$
H(x, y)=\frac{x^{2}}{2}+\frac{y^{2}}{2}+\frac{y^{4}}{4}=h \in(0,+\infty)
$$

we obtain that

$$
L^{ \pm}(h): x= \pm \sqrt{2 h-y^{2}-\frac{y^{4}}{2}}
$$

and

$$
\alpha(h)=\sqrt{1+h}-1, \quad \alpha_{1}(h)=-\alpha(h) .
$$

According to Theorem 1, the first-order Melnikov function is

$$
\begin{aligned}
M_{1}(h)= & \int_{L_{h}^{+}}\left(\int_{0}^{x} g_{y}^{+}(x, y) \mathrm{d} x+f^{+}(x, y)\right) \mathrm{d} y \\
& +\int_{L_{h}^{-}}\left(\int_{0}^{x} g_{y}^{-}(x, y) \mathrm{d} x+f^{-}(x, y)\right) \mathrm{d} y \\
= & \int_{L_{h}^{+}}\left(\int_{0}^{x} g_{y}^{+}(x, y) \mathrm{d} x+f^{+}(x, y)\right) \mathrm{d} y \\
& -\int_{L_{h}^{+}}\left(\int_{0}^{-x} g_{y}^{-}(x, y) \mathrm{d} x+f^{-}(-x, y)\right) \mathrm{d} y,
\end{aligned}
$$

where $f^{ \pm}(x, y)$ and $g^{ \pm}(x, y)$ are given by (5).

For simplicity, we define the following functions:

$$
I_{i, j}(h)=\int_{L_{h}^{+}} x^{i} y^{j} \mathrm{~d} y, \quad h \in(0,+\infty) .
$$

Lemma 7 The following equalities hold: 5

(i) $I_{0,1}(h)=I_{1,1}(h)=I_{2,1}(h)=I_{0,3}(h)=0$;

(ii) $I_{0,0}(h), I_{1,0}(h), I_{2,0}(h), I_{0,2}(h), I_{3,0}(h), I_{1,2}(h)$ are linearly independent functions. 
Proof (i) Note that $L_{h}^{+}: x=\sqrt{2 h-y^{2}-\frac{y^{4}}{2}}$ and $\alpha_{1}(h)=-\alpha(h)$. By symmetry we have

$$
I_{0,1}(h)=\int_{L_{h}^{+}} y \mathrm{~d} y=\int_{\alpha(h)}^{-\alpha(h)} y \mathrm{~d} y=0 .
$$

The equalities $I_{1,1}(h)=I_{2,1}(h)=I_{0,3}(h)=0$ can be proved similarly.

(ii) In order to prove that $I_{0,0}(h), I_{1,0}(h), I_{2,0}(h), I_{0,2}(h), I_{3,0}(h), I_{1,2}(h)$ are linearly independent functions, for these functions, we make the following Taylor expansions in the variable $h$ around $h=0$ :

$$
\begin{aligned}
& I_{0,0}(h)=-h+\frac{1}{4} h^{2}-\frac{1}{8} h^{3}+O\left(h^{4}\right), \\
& I_{1,0}(h)=-\sqrt{2} h^{\frac{3}{2}}+\frac{13 \sqrt{2}}{48} h^{\frac{5}{2}}-\frac{359 \sqrt{2}}{2,560} h^{\frac{7}{2}}+O\left(h^{\frac{9}{2}}\right), \\
& I_{2,0}(h)=-2 h^{2}+\frac{7}{12} h^{3}+O\left(h^{4}\right), \\
& I_{3,0}(h)=-2 \sqrt{2} h^{\frac{5}{2}}+\frac{5 \sqrt{2}}{8} h^{\frac{7}{2}}+O\left(h^{\frac{9}{2}}\right), \\
& I_{0,2}(h)=-\frac{1}{12} h^{3}+O\left(h^{4}\right), \\
& I_{1,2}(h)=-\frac{\sqrt{2}}{12} h^{\frac{7}{2}}+O\left(h^{\frac{9}{2}}\right) .
\end{aligned}
$$

Suppose that

$$
F(h)=k_{1} I_{0,0}(h)+k_{2} I_{1,0}(h)+k_{3} I_{2,0}(h)+k_{4} I_{0,2}(h)+k_{5} I_{3,0}(h)+k_{6} I_{1,2}(h) \equiv 0 .
$$

In the following, we need to prove that $k_{i}=0, i=1,2, \ldots, 6$.

From $F^{\prime}(0)=-k_{1}=0$ we have $k_{1}=0$. Substituting $k_{1}=0$ into $(21)$, from $\lim _{h \rightarrow 0^{+}} \frac{F(h)}{h^{\frac{3}{2}}}=$ $-\sqrt{2} k_{2}=0$ we have $k_{2}=0$. Substituting $k_{1}=k_{2}=0$ into (21), from $F^{\prime \prime}(0)=-4 k_{3}=0$ we have $k_{3}=0$. Similarly, we can obtain that $k_{4}=k_{5}=k_{6}=0$.

Substituting statement (i) of Lemma 7 into (17), we have

$$
\begin{aligned}
M_{1}(h)= & \left(c_{0,0}-a_{0,0}\right) I_{0,0}(h) \\
& -\left(a_{1,0}+b_{0,1}+c_{1,0}+d_{0,1}\right) I_{1,0}(h) \\
& -\left(a_{2,0}-c_{2,0}+\frac{b_{1,1}}{2}-\frac{d_{1,1}}{2}\right) I_{2,0}(h) \\
& +\left(c_{0,2}-a_{0,2}\right) I_{0,2}(h) \\
& -\left(a_{3,0}+c_{3,0}+\frac{b_{2,1}}{3}+\frac{d_{2,1}}{3}\right) I_{3,0}(h) \\
& -\left(a_{1,2}+3 b_{0,3}+c_{1,2}+3 d_{0,3}\right) I_{1,2}(h) .
\end{aligned}
$$

By statement (ii) of Lemma 7 the first-order Melnikov function $M_{1}(h)$ given by $(21)$ is a linear combination of six linearly independent functions $I_{0,0}(h), I_{1,0}(h), I_{2,0}(h), I_{0,2}(h), I_{3,0}(h)$, 
$I_{1,2}(h)$ with arbitrary coefficients. From Lemma 6 we obtain that $M_{1}(h)$ has at least five simple zeros in $(0,+\infty)$. According to Theorem 1 , we can deduce that there are at least five limit cycles that can bifurcate from the period annulus surrounding the origin of (3) by the first-order Melnikov function.

The proof of Theorem 3 for system (4) is similar. The main difference is that

$$
L^{ \pm}(h): x= \pm \sqrt{2 h-y^{2}+\frac{y^{4}}{2}}, \quad h \in\left(0, \frac{1}{4}\right)
$$

and

$$
\alpha(h)=\sqrt{1-\sqrt{1-4 h}}, \quad \alpha_{1}(h)=-\alpha(h),
$$

so we omit it.

Competing interests

The authors declare that they have no competing interests.

\section{Authors' contributions}

Both authors contributed equally in this article. They read and approved the final manuscript.

\section{Author details}

'Department of Mathematics, Sichuan University, Chengdu, 610064, P.R. China. ${ }^{2}$ Department of Mathematics, Guizhou University, Guiyang, 550025, P.R. China. ${ }^{3}$ School of Mathematics and Statistics, Guangdong University of Finance and Economics, Guangzhou, 510320, P.R. China.

\section{Acknowledgements}

The authors would like to thank the editor and the anonymous reviewers for their useful and valuable suggestions. This work is supported by the National Natural Science Foundation of China (Nos. 11301105, 11401111) and Science Technology fund of Foundation Guizhou Province (No. [2015]2036), Guizhou Science Foundation for Distinguished Young Scholars (201313), and Science and Technical Innovative Personnel Foundation of Guizhou Education Department (2012085).

Received: 15 June 2016 Accepted: 26 August 2016 Published online: 01 September 2016

\section{References}

1. Gavrilov, L: The infinitesimal 16th Hilbert problem in the quadratic case. Invent. Math. 143, 449-497 (2001)

2. Li, C, Li, W: Weak Hilbert's 16th problem and the relative research. Adv. Math. 39, 513-526 (2010)

3. Li, J: Hilbert's 16th problem and bifurcations of planar polynomial vector fields. Int. J. Bifurc. Chaos 13, 47-106 (2003)

4. Xiong, Y, Han, M, Xiao, D: New lower bounds for the Hilbert number of polynomial systems of Liénard type. J. Differ. Equ. 257, 2565-2590 (2014)

5. Smale, S: Mathematical problems for the next century. Math. Intell. 20, 7-15 (1998)

6. Banerjee, S, Verghese, G: Nonlinear Phenomena in Power Electronics: Attractors, Bifurcations, Chaos, and Nonlinear Control. Wiley-IEEE Press, New York (2001)

7. di Bernardo, M, et al.: Bifurcations in nonsmooth dynamic systems. SIAM Rev. 50, 629-701 (2008)

8. Martins, R, Mereu, A: Limit cycles in discontinuous classical Liénard equations. Nonlinear Anal., Real World Appl. 20, 67-73 (2014)

9. Llibre, J, Teixeira, M: Limit cycles for m-piecewise discontinuous polynomial Liénard differential equations. Z. Angew. Math. Phys. (2014). doi:10.1007/s00033-013-0393-2

10. Yang, J, Han, M, Huang, W: On Hopf bifurcations of piecewise planar Hamiltonian systems. J. Differ. Equ. 250, 1026-1051 (2011)

11. Liu, X, Han, M: Bifurcation of limit cycles by perturbing piecewise Hamiltonian systems. Int. J. Bifurc. Chaos 20 1379-1390 (2010)

12. Xiong, Y: Limit cycle bifurcations by perturbing piecewise smooth Hamiltonian systems with multiple parameters J. Math. Anal. Appl. 421, 260-275 (2015)

13. Llibre, J, Mereu, A: Limit cycles for discontinuous quadratic differential systems with two zones. J. Math. Anal. Appl. 413, 763-775 (2014)

14. Giacomini, H, Llibre, J, Viano, M: On the nonexistence, existence and uniqueness of limit cycles. Nonlinearity 9 , 501-516 (1996)

15. Li, C, Llibre, J: A unified study on the cyclicity of period annulus of the reversible quadratic Hamiltonian systems. J. Dyn. Differ. Equ. 16, 271-295 (2004)

16. Zhao, Y, Zhang, Z: Linear estimate of the number of zeros of Abelian integrals for a kind of quartic Hamiltonians. J. Differ. Equ. 155, 73-88 (1999) 
17. Gavrilov, L, lliev, ID: Complete hyperelliptic integrals of the first kind and their non-oscillation. Trans. Am. Math. Soc. 356, 1185-1207 (2004)

18. Wang, J, Xiao, D: On the number of limit cycles in small perturbations of a class of hyper-elliptic Hamiltonian systems with one nilpotent saddle. J. Differ. Equ. 250, 2227-2243 (2011)

19. Xiong, Y: Bifurcation of limit cycles by perturbing a class of hyper-elliptic Hamiltonian systems of degree five. J. Math. Anal. Appl. 411, 559-573 (2014)

20. Iliev, ID, Perko, LM: Higher order bifurcations of limit cycles. J. Differ. Equ. 154, 339-363 (1999)

21. Coll, B, Gasull, A, Prohens, R: Bifurcation of limit cycles from two families of centers. Dyn. Contin. Discrete Impuls. Syst., Ser. A Math. Anal. 12, 275-287 (2005)

Submit your manuscript to a SpringerOpen ${ }^{\odot}$ journal and benefit from:

- Convenient online submission

Rigorous peer review

- Immediate publication on acceptance

- Open access: articles freely available online

- High visibility within the field

- Retaining the copyright to your article 